\title{
Faces of Twins
}

\author{
Nancy L. Segal \\ Department of Psychology, California State University, USA \\ (This article is dedicated to my uncle, Dr. Allan Rubenstein, in fond memory.)
}

There is great visual interest in monozygotic (MZ) co-twins' near identity in face and form. Behind their matching faces is a world of information that psychologists, anthropologists, physicians and others are exploring via sophisticated new theories and techniques. The findings have implications for informationprocessing, psychosocial adjustment, personal identification and much more. The research reviewed below samples from a wide range of scientific disciplines and domains. We learn that facial variation prevails despite MZ twins' shared genes, and that appearances can be deceptive.

This "facial tour" begins with an intriguing study conducted by British psychologist Sarah V. Stevenage (1998). Noting that MZ twins' faces are among "the most perceptually challenging stimuli we encounter" (p. 39), a set of experiments is described that elucidate the path from confusion to clarity. The aim of this study was to induce categorical perception in the viewer - categorical perception occurs when the category membership of a stimulus affects its perception. There is evidence that humans categorize naturally occurring or highly familiar phenomena such as colors, sounds and facial expressions; the first two (colors and sounds) are thought to engage "low level" processes, while the latter (facial expressivity) is thought to engage "high level" processes. However, categorical perception may not occur when stimuli fall into unnatural or atypical categories. (I suspect that MZ twins' similar faces can be considered exceptional, albeit naturally occurring, phenomena.) It was expected that people could be trained to distinguish between $M Z$ twins, that is, learn to recognize that co-twins are members of different categories.

First, participants provided similarity ratings for a series of photographs paired in various combinations of Twin $A-T w i n A$, Twin B Twin B and Twin A - Twin B. Next, they indicated whether individual photographs presented in blocks depicted Twin A or Twin B. They were immediately informed of the accuracy of each answer, a process that continued until a minimum accuracy rate of $90 \%$ was achieved across two consecutive blocks. A second set of similarity ratings for photographs was then obtained following this procedure. Individual differences in participants' learning curves were observed. Nevertheless, it was concluded that induction of categorical perception was successful because similarity ratings of Twin A Twin A and Twin B - Twin B pairings increased and similarity ratings of Twin A - Twin B pairings decreased.

There is experimental evidence that ability to categorize new stimuli may be affected by previous exposure to similar stimuli. A second experiment was, therefore, conducted to determine if categorization of twins in the first MZ set influenced categorization of twins from a second set. The same procedure was followed, with the exception that respondents indicated whether photographs were "same" or "different" and estimated their confidence in their answers. In this case, learning curves suggested improvement to the point at which co-twins could be visually discriminated, but not always correctly identified. Following several errors, improvement increased gradually. A two-stage learning process was, thus suggested: (1) learning to differentiate the twins and (2) learning to label the twins.

It is reasonable to suppose that telling twins apart in everyday life follows a similar plan. Many people meeting twins for the first time focus initially on finding identifying features. Once this is accomplished, they associate these "markers" with one twin and not the other. In contrast, MZ twins' parents (especially mothers) and siblings have little difficulty in telling twins apart. This observation is viewed with wonder by those still struggling, but the categorical perception experiments may explain why: Parents and siblings may master categorical induction before everyone else.

What makes twins easy or difficult to tell apart most likely varies from pair to pair. Computer-manipulated images of $\mathrm{MZ}$ twins may be helpful in this regard (Bruce \& Young, 1994). These photographs exaggerate twins' facial similarities and differences, thus revealing why we confuse twins, but also why we may eventually tell them apart.

Twins' facial features are not limited to what we can see. Similarities in MZ twins' facial structures or contours mask a deeper difference: the patterning of blood vessels. These unique configurations lying beneath the surface of everyone's visage can be visualized via a thermogram (Ross, 1994). Thermograms are images created when an infrared camera captures the heat radiated from a person's face. Their use in personal identification was first proposed by Francine 
Prokovski in the 1970s, an idea she developed later on. Just as MZ twins lack precisely matching fingerprints, they lack completely coincident blood vessel networks.

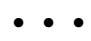

Just as some MZ twins look very much alike, a minority look very different from one another. Some of these pairs are part of the rare twin group discordant for hemifacial microsomia (HFM). HFM is a craniofacial anomaly (CFA) estimated to affect children in 1 in 5600 births (Maris et al., 1999). Its major effects are altered development of the eyes, ears and jaw, although expression is variable. Most studies assessing psychosocial adjustment of affected individuals have used samples representing a variety of facial malformations. This is unfortunate because heterogeneous groupings can conceal effects unique to HFM. In an effort to correct this gap, a multidisciplinary team at the University of Washington in Seattle conducted an extensive behavioral investigation of 12 children, focusing specifically on HFA (Maris et al., 1999). The children consisted of six boys and six girls between the ages of 9 and 15 years. They were members of six same-sex twin pairs.

The twins were patients at a dental clinic in a large children's hospital. Parents completed the Child Behavior Checklist (CBCL) and teachers completed the corresponding Teacher Report Form (TRF). Children responded to items on the Coopersmith Self-Esteem Inventory and to a modified version of the SelfInterview. Data analysis showed that HFM twins had a significantly higher frequency of behavioral problems than their unaffected co-twins, as rated by parents and teachers. In contrast, co-twins' CBCL competence scores did not differ; closer inspection revealed that HFM twins scored especially high in the activities section. The investigators speculated that affected twins might be compensating for behavioral problems in other domains. (Comparable competence data from the TRF were unavailable.) Self-esteem scores were higher for unaffected twins, as expected, but scores of all twins were in the normal range. Interviews revealed that HFM twins were concerned with their appearance, adverse reactions from others and medical procedures. Unaffected twins worried over the effects of HFM on their co-twins, described their co-twins as shy, and showed understanding of their co-twins' emotional circumstances.

The investigators noted that "previous psychosocial research on children with HFM leaves unanswered the question of how children with HFM fare relative to children without CFAs" (p. 45). They were clearly aware of how well suited their unique sample was for addressing this issue and should be commended for undertaking this important study. For example, naturally occurring cotwin control studies afford better behavioral comparisons between affected and non-affected children than would be possible using nontwin samples. The sample also presented the unusual opportunity to study the effects of living with someone "who represented how they might have looked, had they not been born with HFM" (Maris et al., p. 45).

Regrettably, zygosity determinations were not made in this study. This decision was explained by the investigators' interest in comparing behaviors in HFM and non-HFM individuals with matching background variables, not with discerning genetic and environmental effects. However, failure to consider twin type clouds interpretation of the findings. This is because differences between DZ co-twins included in the study might have reflected differences in intelligence, personality and/or temperament, as well as differences in disease status. Knowledge of zygosity would not have yielded definitive findings from such a small sample, but this information would have enabled pooling of these pairs with others as they become identified (Segal \& Bouchard, 1984).

Longitudinal assessment (ages 12 to 18 years) of the facial growth of a set of MZ female co-twins discordant for congenital heart disease has also revealed striking differences in facial morphology (Burke \& Hughes, 1987). Early physical differences between the twins were observed as early as 10 months of age, although yearly observations did not commence until they were twelve. In addition to standard height and weight measurements, the development of soft facial tissues was assessed via three-dimensional stereophotogrammetry. This procedure avoids distortions posed by the use of calipers and two-dimensional photography. It involves analysis of various linear facial parameters, each defined by anatomical landmarks. Thirteen non-overlapping parameters were chosen for this study. The zygosity of the pair was determined by bloodtyping studies.

The healthy twin had a larger face during the six-year course of the study. The degree of co-twin difference was not constant, but decreased between ages 13 and 15 and between ages 16 and 18 during periods of rapid growth. Many individual growth parameters, such as mouth width, nasal height and posterior facial height, followed this pattern. In contrast, some parameters, such as nose tip to upper lip, were closely matched between the twins. Lip height decreased for both twins, a change that often co-occurs with alteration in lip posture during adolescence. Another developmental measure of interest was the ratio of facial height to facial width. The typical pattern for female children is larger facial width until age ten, followed by larger facial height during later development. It was anticipated that this ratio might furnish an approximate index of facial development in these twins even though they had passed the ten-year mark. The healthy twin had a relatively larger facial height at age twelve, in contrast with her twin sister whose relatively greater facial width was thought to reflect delayed facial growth. This ratio eventually reversed as the affected twin approached late adolescence.

The investigators emphasized that visual inspection of twins' facial morphology is inappropriate for diagnosing zygosity. The twins in this study were discordant for a major medical condition, but facial differences could also be a legacy of $\mathrm{MZ}$ twins' pre and perinatal circumstances. Even while the zygosity of 
many twins can be correctly determined by responses to simple questions (e.g., Are you as alike as two peas in a pod?), not all peas are precise duplicates and some that seem so may hide essential discrepancies.

$$
\text { -・・ }
$$

Finally, MZ twins' faces may be windows into features associated with human reproductive fitness. This is illustrated by the creative efforts of Australian researchers who linked MZ twins, facial appearance (physical attractiveness, specifically) and evolutionary psychology (Mealey et al., 1999). The study was a test of fluctuating asymmetry (FA), defined as departure from left-to-right body consistency. FA has been assessed by sidedness differences in wrist circumference, facial width, ear length and other measures. Greater FA is believed to reflect developmental instability associated with stressful biological and environmental events. Greater body symmetry (reduced FA) is an established correlate of physical attractiveness. A recent controversy has been the extent to which FA may be a marker of reduced biological fitness (ability to produce viable children). Studies concerned with relationships between physical attractiveness and body symmetry have not, however, controlled for various factors linked to attractiveness, such as age and skin tone - until now.

The research team capitalized on the availability of standardized photographs of $\mathrm{MZ}$ twins to further explore physical attractiveness - facial symmetry relationships. Photographs were cut and reconstructed to obtain Left-Left and Right-Right composites of each co-twin. Judges indicated if the two facial composites were more similar for Twin 1 or for Twin 2 . This response provided the measure of which co-twin was more symmetric/asymmetric. Judges also rated the similarity of each Left-Left and RightRight composite. This rating provided an index of facial symmetry between pairs. A different panel of judges then assessed the physical attractiveness of each individual twin, relative to his or her co-twin. Major findings were that (1) the more symmetric twin was viewed as more attractive physically and (2) the size of the co-twin difference in physical attractiveness was related to the size of the difference in facial symmetry. These findings pave the way for further analyses along these lines. It would, for example, be important to know if the less symmetric $\mathrm{MZ}$ co-twin were less healthy, less likely to reproduce and/or less successful in other life history domains relevant to biological fitness.

\section{Research Reviews}

\section{Male-Female Twins in Utero}

There has been renewed interest in male-female twins as a model for determining if, and how, prenatal exposure to cross-sex hormones affects physical and behavioral development. This interest has been inspired by a wealth of findings from the nonhuman literature. Studies of mice (Kinsley et al., 1986) and gerbils (Clark \& Galef, 1994) have reported increased masculinization of the behaviors of females positioned between male fetuses in utero. Other evidence comes from opposite-sex cattle twins that display the wellknown freemartin effect in which female co-twins become sterile (Miller, 1994). Findings are mixed with respect to humans, but the weight of evidence does not appear supportive.

Most behavioral researchers are, however, aware of the difficulty in disentangling possible prenatal hormonal influences from social-interactional effects. Elsewhere, I have suggested that this might be accomplished by contrasting the behaviors of same-age opposite-sex unrelated siblings (children adopted as infants into the same family who share rearing environments, but not prenatal environments) with opposite-sex twins reared apart from birth (twins adopted separately as infants, who share prenatal environments, but not rearing environments) (Segal, 2000). The only research more fully supportive of the prenatal hormonal exposure hypothesis found reduced frequency of spontaneous otoacoustic emissions (SPOEs: continuous tones in the inner ear intended to amplify the volume of weak sounds to make them audible) in females with twin brothers, relative to other female twins and non-twins (McFadden, 1993). Female twins did not differ in SPOE frequency relative to their twin brothers. This pattern of findings is difficult to reconcile with reference to the twins' social relations or rearing.

Recent work by Danish investigators has extended this work (Gaist et al., 2000). Middle-aged twins completed tests of handgrip strength, height, weight, body mass index and weight circumference. Values were comparable among DZ same-sex, DZ opposite-sex and $M Z$ twin pairs, thus contributing to the body of non-supportive evidence.

\section{Transsexualism in MZ Female Twins}

MZ twin concordance for a rare behavior may encourage researchers to rethink current explanations. Female transsexualism is estimated to occur in approximately 1 in 100, 000 women; thus, the identification of an eighteen-year-old apparently MZ female set concordant for this condition was of interest (Sadeghi \& Fakhrai, 2000). (The twins' zygosity was based on their identical appearance, but was not confirmed by objective testing.) The first twin who drew medical attention to the case indicated a desire for male activities and attire beginning at age three. In her social relations with other females she fantasized the role of male dating partner. She believed she was destined to have been male and requested surgery for 